\title{
Uso de um software de correção automática: uma al- ternativa para viabilizar o processo avaliativo contí- nuo no contexto do ensino universitário
}

\section{Use of automatic correction software: an alternative to allow the process of continuous assessment in the context of university education}

\author{
RICARDO ANDREAS SAUERWEIN \\ Universidade Federal de Santa Maria - UFSM \\ JOSEMAR ALVES \\ Universidade Federal de Santa Maria - UFSM \\ DIONI PAULO PASTORIO
}

Universidade Federal do Rio Grande do Sul - UFRGS

\begin{abstract}
Resumo: A avaliação continuada tem sido apontada como um dos fatores-chave para se alcançar uma aprendizagem mais efetiva. Porém, no caso de turmas grandes, um limitante para a implementação desse tipo de avaliação é a sobrecarga que acarreta ao docente no que concerne à correção, processamento e divulgação do aproveitamento das atividades realizadas. 0 emprego de ferramentas computacionais para apoiar o trabalho docente no processo avaliativo vem sendo apontado como uma das possíveis alternativas para superar essa dificuldade. Por isso, neste trabalho, temos por objetivo investigar as potencialidades de um software de correção automática - Auto Multiple Choice (AMC). Ou seja, analisamos o AMC como ferramenta usada na implementação de um processo de avaliação continuada, baseada na resolução de problemas numéricos, composto de seis (6) testes curtos e duas (2) provas, o qual foi implementado com duas turmas de cursos Engenharia, com um total de sessenta e sete (67) alunos.
\end{abstract}

Palavras-chave: Software de correção automática. Auto Multiple Choice. Processo avaliativo contínuo. Ensino de Física.

Abstract: Continuous assessment has been identified as one of the key factors for achieving more effective learning. However, in the context of large classes, a limitation for the execution of this type of assessment is the overload that it entails to the teacher regarding the correction, processing and feedback of the activities carried out. The use of computational tools to support the teacher work in the assessment process has been pointed out as one of the possible alternatives to overcome this difficulty. Therefore, in this work, we aim to investigate the potentialities of an automatic correction software - Auto Multiple Choice (AMC). That is, we analyze the AMC as a tool effectively used in the implementation of a continuous assessment process, based on the solving of numerical problems, composed of 6 short tests and 2 main tests, which was implemented with two classes of engineering courses, with a total of 67 students.

Keywords: Automatic correction software. Auto Multiple Choice. Continuous assessment process. Physics teaching. 


\section{Introdução}

Muitas pesquisas têm apontado que, para se alcançar uma aprendizagem mais efetiva ${ }^{1}$, é preciso desenvolver um processo avaliativo contínuo (algumas vezes denominado de formativo), o qual forneça feedbacks rápidos (em tempo hábil para contribuir com a aprendizagem do aluno) e frequentes para os estudantes ao longo do desenvolvimento de todo o processo de ensino e aprendizagem (RAVITZ, 2000; OTSUKA et al., 2003; MAXIMO et al., 2007; VITTURINI et al., 2011; PÉREZ-BENEDITO et al., 2014).

Conforme destacam Pérez-Benedito et al. (2014), avaliar os estudantes dessa maneira desempenha um papel fundamental no processo de ensino e aprendizagem tanto na perspectiva do professor, quanto na do aluno. Isso porque um processo avaliativo como o mencionado anteriormente fornece subsídios aos estudantes, de modo que eles possam ter maior consciência e controle sobre suas próprias aprendizagens (OLIVEIRA, 2002; RAVITZ, 2000; OTSUKA et al., 2003; PÉREZ-BENEDITO et al., 2014). Paralelamente, provê importantes subsídios ao trabalho docente, de maneira que o professor possa acompanhar com maior eficiência o desenvolvimento geral e individual de seus alunos, assim como avaliar quais mudanças ou correções ele precisa efetuar nas atividades que vêm sendo executadas em sua disciplina para alcançar os objetivos de aprendizagem almejados inicialmente (OLIVEIRA, 2002; OTSUKA et al., 2003; VITTURINI et al., 2011; PÉREZ-BENEDITO et al., 2014).

No entanto, no atual contexto brasileiro de ensino de física (em particular nas disciplinas básicas do ensino superior) o processo avaliativo continua sendo constituído principalmente por duas ou três avaliações (normalmente provas ou testes) pontuais e cumulativas, as quais dividem o conteúdo em grandes partes e são, em geral, compostas por problemas como os presentes nos finais de capítulos de livros didáticos ou ainda, exercícios de reprodução de equações prontas e definidas.

Cabe destacar, contudo, que o uso de problemas desse tipo nas avaliações não é (isoladamente) o que torna o processo avaliativo deficiente no sentido de promover um processo de ensino e aprendizagem mais efetivo. Isto é, uma vez que a resolução de problemas já está amplamente reconhecida como importante estratégia didática e vem sendo empregada corriqueiramente em salas de aula de física (CLEMENT; TERRAZZAN, 2012), nada mais coerente de que os docentes a empregarem como um dos instrumentos avaliativos. Por outro lado, realizar apenas avaliações pontuais e cumulativas, assim como fornecer feedbacks também pontuais, ou ainda, muito lentos e sem conexão com as atividades, podem ser dois fatores preponderantes para que esse processo seja ineficiente (consoante ao que vem sendo apontado na literatura).

Uma possível explicação para o uso disseminado de avaliações pontuais e cumulativas nesse contexto de ensino - ainda que trabalhos presentes na literatura apontem no sentido da avaliação continuada - pode ser o fato de que a quantidade de estudantes atendidos em disciplinas universitárias de Física Básica, conforme evidencia Mello (2015), vem aumentado consideravelmente nos últimos anos. É comum, a maioria das salas de aula no contexto universitário, contar com mais de cinquenta/sessenta alunos nas classes. Logo, desenvolver um processo avaliativo contínuo torna-se pouco viável nesse contexto, uma vez que o processo de correção e feedback desse formato de avaliação seria bastante demorado para o docente (OTSUKA; DA ROCHA, 2005).

Assim, segundo indícios apontados por Otsuka et al. (2003), Pérez-Benedito et al. (2014) e Mello (2015), desenvolver um processo avaliativo contínuo e, ao mesmo tempo, viável no contexto do processo de ensino e aprendizagem (em especial no ensino universitário) constitui-se

1 Ou, em outras palavras, um processo de ensino e aprendizagem mais eficiente e produtivo em que os objetivos de aprendizagem propostos tenham maior possibilidade de serem alcançados pelos estudantes. 
em um dos desafios para a pesquisa em ensino em geral e de física em particular. Portanto, uma importante questão didática no âmbito do ensino de física é: como viabilizar o desenvolvimento de um processo avaliativo contínuo nas disciplinas de Física Básica no contexto universitário atual?

Uma das possíveis formas, que vem sendo apontada na literatura, de solucionar a dificuldade acima referida é empregar ferramentas computacionais para apoiar o trabalho docente durante o processo avaliativo (RAVITZ, 2000; OTSUKA et al., 2003; VITTURINI et al., 2011; MAXIMO et al., 2007; PÉREZ-BENEDITO et al., 2014; MELLO, 2015). Em particular, neste trabalho, propomos que usar essas ferramentas no sentido de dinamizar a etapa de correção e feedback das avaliações desenvolvidas pode ser um dos passos fundamentais para viabilizar a realização de um processo avaliativo formativo nas disciplinas de física básica no já referido contexto de ensino.

Somado a isso, a experiência docente de um dos autores deste trabalho nos convenceu sobre a necessidade de implementar um sistema de avaliação continuada. Nesse sentido, sempre que possível, utilizamos um sistema composto de 6 a 8 testes e 2 provas nas disciplinas que ministramos. Esses testes são formados, em geral, por uma única questão (aqui, nesse contexto, entendido como problema) a ser resolvida em cerca de 20 a 25 minutos. Já as provas são compostas por 4 ou 5 questões e devem ser resolvidas em cerca de 100 a 120 minutos. As questões das provas são constituídas por problemas do mesmo nível (mas não iguais) aos dos testes 2 .

Contudo, em nossa experiência prévia com turmas grandes ${ }^{3}$, na maioria dos casos não era possível implementar um sistema de avaliação continuada por limitações de ordem prática. Por exemplo, um dos problemas apresentado estava ligado à aplicabilidade dos testes: o sistema avaliativo que utilizamos exige que esses testes sejam resolvidos individualmente (em uma condição semelhante à das provas). Porém, entendemos que a situação de aplicação de prova em que o professor toma medidas adicionais para garantir resoluções de provas independentes e sem consultas seja aceitável apenas pontualmente. Ou seja, acreditamos que o caráter de supervisão permanente dessa situação é viável se feita duas vezes ao longo de um semestre, mas que, em uma periodicidade quase semanal, torna-se impraticável. Outro problema que inviabilizava a implementação de um sistema avaliativo continuado em turmas grandes era o tempo necessário para corrigir as avaliações aplicadas. Uma vez que as turmas eram compostas por um grande número de alunos, como já frisamos, o processo de feedback se tornava muito lento e, por vezes, impossível de ser realizado.

Em vista disso, neste trabalho, apresentamos como o AMC foi utilizado para implementar o sistema de avaliação continuada em turmas de Física Geral de Engenharias, as quais contavam com um grande número de alunos e que sem esse recurso não poderiam ter se beneficiado deste tipo de avaliação. Além disso, acreditamos que ferramentas como o AMC vêm sendo pouco abordadas na literatura da área, bem como que o uso dessas ferramentas permite implementar processos de avaliação continuada que têm inegáveis impactos na qualificação do processo de ensino e aprendizagem. Portanto, entendemos que é preciso discutir suas principais características, potencialidades e limitações, o que é feito neste trabalho através da análise do uso do AMC durante sua utilização na implementação de um processo de avaliação continuada.

\footnotetext{
2 Cabe destacar que na maioria das vezes, os problemas eram retirados ou adaptados do livro texto referência da disciplina (Halliday).

3 Estamos, aqui, relacionando esse argumento com outras atividades didáticas, de diferentes naturezas, realizadas durante o curso de pós-graduação e as atividades de sala de aula dos autores.
} 


\section{Software Auto Multiple Choice (AMC)}

O AMC é um software livre e gratuito, disponibilizado sob licença GNU, que implementa um sistema computacional para preparação, gerenciamento e avaliação (correção) de testes de múltipla escolha. Esse aplicativo possui uma interface gráfica amigável e documentação detaIhada sobre os recursos oferecidos e como utilizá-los. O objetivo desta seção não é substituir esta documentação oficial4, mas sim apresentar uma breve descrição do AMC, que informe a respeito de seus aspectos básicos. Assim, acreditamos que o leitor terá mais elementos para acompanhar a implementação que realizamos com o objetivo de investigar as potencialidades do AMC, bem como poderá decidir em que medida esse aplicativo pode vir a atender suas próprias necessidades.

O sistema AMC foi desenvolvido em torno de sua capacidade de reconhecimento ótico de imagens digitalizadas de testes de múltipla escolha, logo, ele é uma ferramenta particularmente útil para a implementação de avaliações sistemáticas presenciais, uma vez que, após elaboradas, as etapas de correção, atribuição de nota e divulgação dos resultados são automatizadas. A sequência de trabalho típica de um teste AMC envolve as seguintes etapas:

(1) Preparação;

(2) Impressão de folhas de questões e de respostas (ou folhas mistas);

(3) Implementação;

(4) Digitalização das folhas com as respostas;

(5) Processamento.

$\mathrm{Na}$ fase de preparação, o professor deve escrever as questões que vão compor o teste usando as especificações do próprio AMC, pois somente assim o documento digitalizado será reconhecido (processável) pelo sistema nas etapas posteriores. Se o professor já tem questões prontas, ele deve adaptá-las usando uma linguagem padronizada bastante intuitiva chamada AMC-TXT. Essa linguagem define uma sintaxe simples através da qual se insere no próprio documento da questão o seu gabarito, valor/nota e critérios de correção. As questões podem ser dos seguintes tipos: (a) de escolha única; (b) de escolha múltipla; (c) de escolha numérica; e (d) questão aberta. Em testes mais complexos - como os que usam banco de questões, muitas fórmulas matemáticas, tabelas ou outros recursos - o professor deve elaborar suas questões utilizando diretamente a linguagem $L a T e X^{5} \mathrm{em}$ associação com o sistema de macros específicos definidos pelo sistema AMC para essa linguagem.

Figure 1 - Exemplos de questões de escolha de opções do AMC: item 1 de escolha única; e item 2 de escolha múltipla

1 Campo Grande é a capital de que estado brasileiro?

$\begin{array}{llllllll}\square & \text { AM } \quad \square & \text { PR } \quad \square & \text { MS } \quad \square & \text { MT } \quad \square & \text { RR }\end{array}$

24. Quais dos seguintes estados pertencem à região sul?
$\mathrm{AL}$
$\mathrm{PE}$
$\mathrm{PR}$
RS
SP

\footnotetext{
${ }^{4}$ Que pode ser acessada em: http://project.auto-multiple-choice.net/projects/auto-multiple-choice/.

5 LaTex é uma linguagem de marcação de textos bastante poderosa e difundida (principalmente na comunidade científica). Internamente, o AMC processa a linguagem "nativa" AMC-TXT e as transforma em LaTeX e a partir desta obtém o documento final. Logo, devido à integração natural das duas tecnologias, usuários familiarizados com LaTeX conseguem explorar todo o potencial do sistema AMC.
} 
Os dois primeiros tipos de questões mencionadas acima são bastante utilizados nas práticas escolares, no Enem e nas demais provas de ingresso das Universidades, como em testes vestibulares amplamente difundidos no Brasil. Em questões do tipo (a), de escolha única, o aluno deve selecionar apenas uma das alternativas entre várias opções oferecidas (vide Figura 1). Já em questões do tipo (b), também muito usadas em práticas escolares, o aluno se vê de fato com a possibilidade de selecionar uma, várias ou nenhuma das alternativas apresentadas 6 . Um exemplo de questão desse tipo é apresentado na Figura 1.

As questões do tipo (c), de escolha numérica, são bem menos conhecidas e trabalhadas no âmbito do ensino médio e superior, sendo, por isso, melhor explicadas por um exemplo prático. Suponha o seguinte exemplo: qual a razão entre o perímetro e o diâmetro de uma circunferência? A resposta, naturalmente, é $n=3,1415 \ldots$, e usando o AMC esta questão seria vista pelo aluno da forma que é mostrada na Figura 2.

Figure 2 - Exemplos de questões de escolha de escolha numérica

1 Qual a razão entre o perímetro e o diâmetro de uma circunferência?

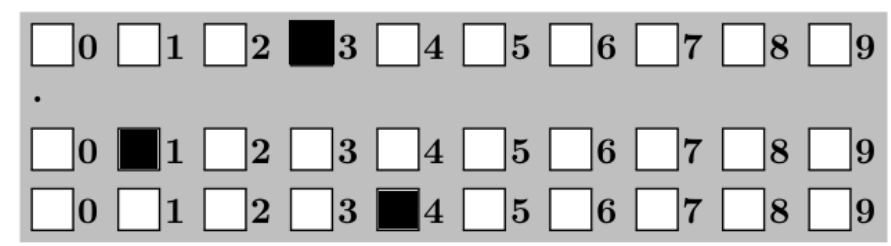

Fonte: elabora pelos autores

Note que há 4 linhas de dados, sendo que um ponto decimal separa as duas primeiras das duas últimas (estamos contando as linhas de cima para baixo). Na convenção adotada pelo AMC, cada linha representa uma casa do sistema decimal que estão dispostas de maneira decrescente de cima para baixo. O ponto indica a posição das linhas das unidades. Desta maneira, na Figura 2, a primeira linha representa a casa das dezenas, a segunda, a das unidades, a terceira, a dos décimos, a quarta, a dos centésimos e assim sucessivamente, sejam quantas forem as linhas abordadas no problema apresentado. Ou seja, as casas dispostas na Figura 2 permitem que o aluno represente qualquer número no intervalo de 0,01 a 99,99. Nas linhas de 1 a 4 foram assinaladas, respectivamente os dígitos $0,3,1$ e 4 , que representam o número decimal 03,14 que é o valor aproximado da razão pedida para a precisão estabelecida implicitamente na questão (representação até a casa dos centésimos). No AMC, o número de casas decimais disponíveis, a posição da marca da unidade (ponto decimal) e, eventualmente, o sinal (positivo ou negativo) podem ser determinados no momento da elaboração da questão.

Nesse tipo de questão, a probabilidade de acerto através de tentativa aleatória, popularmente conhecido como "chute", é muito baixa (no caso da questão exemplo, 1/10000, que é bem menor quando comparado aos $20 \%$ de chances de acertos em questões do tipo múltipla escolha de cinco alternativas). Considerando que erros de arredondamento e numéricos são

\footnotetext{
6 Note que, questões do tipo verdadeiro ou falso, são de fato questões que se enquadram no tipo (b), em que ao assinalar/não assinalar uma alternativa é equivalente a marcá-la como verdadeira/falsa.
} 
toleráveis7, mas devem ser sistematicamente trabalhados, o AMC permite estabelecer dois intervalos em torno do valor gabarito. Assim, é possível atribuir um intervalo em que o resultado marcado seja considerado equivalente ao gabarito e outro no qual o resultado seja considerado uma aproximação razoável, variando o percentual de acerto, de acordo com as necessidades do docente. Por exemplo, na questão acima, poderia ter sido fixada a precisão da resposta equivalente a gabarito em 0,01 e de aproximada em 0,10. Ou seja, as respostas 3,13, 3,14 e 3,15 seriam consideradas gabarito e receberiam o valor integral da questão. Por outro lado, respostas entre 3,04 a 3,24 seriam consideradas aproximadas e poderiam (a critério do professor) receber $80 \%$ do valor da questão, por exemplo. Portanto, esse sistema também permite trabaIhar com os estudantes a questão de precisão numérica e propagação de erros de truncamento.

Por fim, nas questões do tipo (d), que são abertas, o sistema AMC reserva um espaço da página para que o aluno as resolva e, logo acima desse espaço, imprime caixas de seleção reservadas ao professor para que, no momento em que avalia a resposta, atribua um valor, dentre as caixas disponibilizadas pelo AMC, para a solução apresentada. Naturalmente, nesse caso, a correção em si não é automática. Mas considerando um teste com vários tipos de questões, numéricas, de seleções únicas ou múltiplas e abertas, esta facilidade do AMC permite que o professor trabalhe questões abertas e use a automatização implementada pelo AMC para o cálculo da nota total, divulgação dos resultados e o gerenciamento da avaliação.

Cabe destacar ainda, que as questões elaboradas segundo as especificações do AMC podem ser agrupadas em banco de questões. Desta forma, testes posteriores podem ser automaticamente elaborados por meio da inserção de questões lidas do banco. Este recurso viabiliza a parametrização de questões de resposta numérica. Esta estratégia didática consiste em utilizar várias versões da mesma questão, as quais diferem entre si apenas nos dados numéricos de seu enunciado. Em outro trabalho, Alves, Sauerwein e Pastorio (2017), foi discutido a percepção e receptividade desta estratégia em atividades não presenciais disponibilizadas em um ambiente virtual próprio, acessível pela Internet, ou seja, questões escritas no formato eletrônico. Neste trabalho, no entanto, estamos discutindo o uso desse tipo de questão no formato de "lápis e papel", uma vez que o emprego do software AMC viabiliza o uso de questões parametrizadas também em atividades presenciais respondidas em papel.

\subsection{Mais detalhes}

Uma vez elaborados os testes, o professor imprime as folhas de respostas que contêm os campos que serão preenchidos pelos alunos. Nestas folhas, além das questões propriamente ditas, há campos em que o aluno marca seu número de identificação (número na turma, o qual foi previamente cadastrado no sistema juntamente com seu endereço de e-mail, vide Figura 3 ). Dessa forma, após a aplicação dos testes o professor digitaliza as folhas de respostas dos alunos que serão então levadas ao sistema AMC para processamento, o qual é feito nas seguintes etapas:
$>$ 5(a) Captura dos dados;
$>5$ (b) Identificação das seleções realizadas;
$>5$ (c) Identificação dos estudantes;
$>$ 5(d) Exportação dos resultados;
$>$ 5(e) Produção de documentos anotados e envio dos resultados por e-mail.

Cada uma dessas etapas é executada separadamente, para que o professor tenha oportunidade de corrigir manualmente algum engano de leitura do sistema óptico (ou eventual marcação errada/incompleta de algum estudante). Na etapa 5(a), o sistema AMC identifica o docu-

\footnotetext{
7 É perfeitamente compreensível e aceitável que existam variações entre calculadoras e pequenos erros de arredondamento, os quais totalizam variações pontuais no resultado final esperado, que necessariamente precisam ser consideradas na avaliação.
} 
mento digitalizado como um exemplar produzido pelo próprio sistema. Na etapa $5(\mathrm{~b})$, o sistema AMC faz as leituras das marcações feitas pelos alunos. Para leituras bem definidas, quando os campos são preenchidos completamente, o sistema atribui um valor de "qualidade" de leitura próxima a um; em caso contrário, o valor se aproxima de zero. Dessa forma, nesta fase, o sistema AMC apresenta ao professor as seleções nas quais a leitura é duvidosa (ou foi mal marcada pelo aluno), de modo que o professor pode manualmente marcar/desmarcar as seleções de acordo com o que julga ter sido a intenção do aluno.

A etapa $5(c)$ é essencialmente igual à $5(b)$, porém, as marcações se destinam a verificar se o número selecionado pelo aluno confere com algum número previamente cadastrado. Nesta etapa, se o aluno não preencheu corretamente os campos de identificação, o professor tem a oportunidade de atribuir aos testes, pela inspeção visual das assinaturas dos alunos, a identificação do estudante.

$\mathrm{Na}$ etapa 5(d), as notas de aproveitamento nos testes e em cada questão são exportadas para uma planilha, para que o professor possa utilizá-la futuramente. Já na etapa 5(e), as cópias digitalizadas dos alunos são anotadas pelo sistema, que identifica as seleções marcadas pelos estudantes, a seleção gabarito, a nota de aproveitamento em cada questão e a nota de aproveitamento total. Esta etapa também oferece a facilidade de enviar estes documentos por e-mail para que os estudantes tenham um pronto feedback de seus desempenhos no teste.

\section{Exemplo prático de utilização do software AMC}

Conforme já discutimos na introdução desse trabalho, com a finalidade de investigar as potencialidades do software AMC, no sentido de viabilizar um processo avaliativo contínuo, desenvolvemos e implementamos um sistema avaliativo composto por um conjunto de testes presenciais, semanais e duas provas divididas igualmente no semestre (para mais informações, vide a introdução). Mas, por brevidade, neste trabalho, apresentamos apenas os dados relativos aos testes semanais. Esses testes estavam divididos em duas partes: (i) resposta numérica final, a qual era corrigida automaticamente via o software AMC; e (ii) justificativa ou resolução (parte aberta), a qual era corrigida pelo docente, utilizando um software de edição de imagem. Cada uma dessas partes tinha o peso de $50 \%$ da nota total do teste, isso porque entendemos que a resolução desenvolvida pelo estudante é uma parte importante a ser investigada no processo de avaliação, ainda que demande tempo para ser executada (cabe destacar que esse percentual é editável, e o professor define o intervalo que julga adequado). Um exemplar desses testes está representado na Figura 3, abaixo.

Figure 3 - Exemplo de um teste gerado via Software AMC

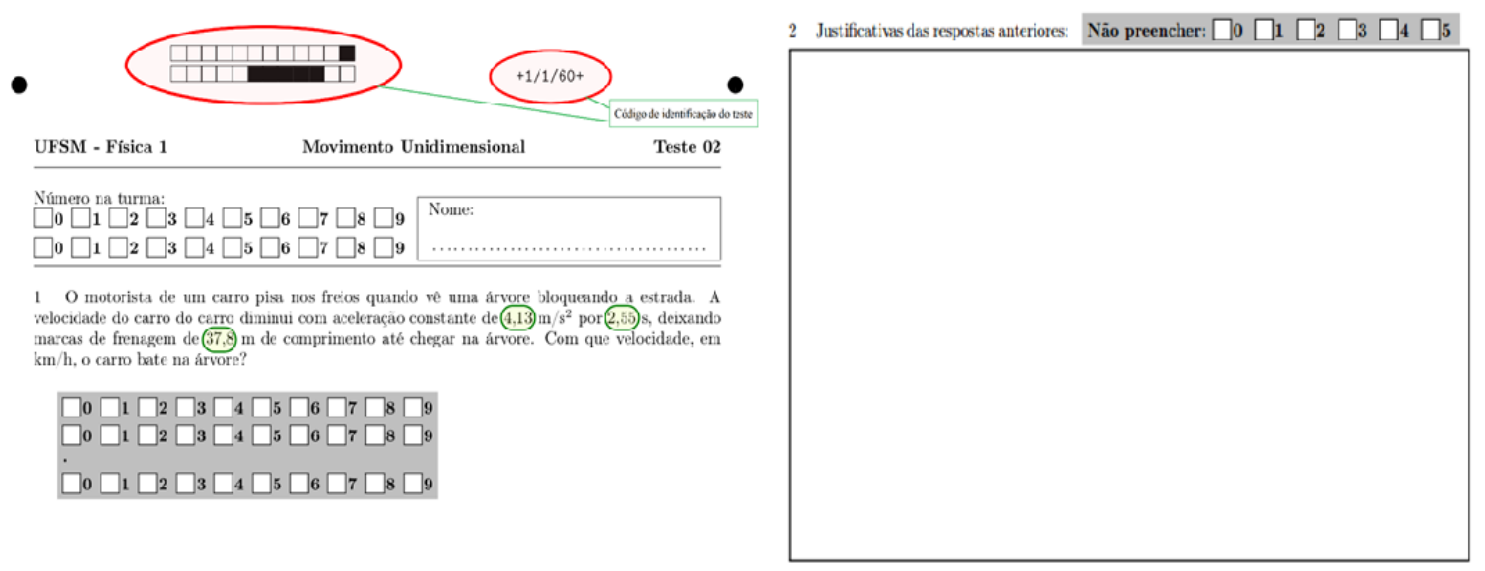


Fonte: elaborada pelos autores

A parte superior de um exemplar desses testes está representada na Figura 3 (para ver uma versão completa desse teste, vide apêndice A). Na referida Figura 3, destacamos:

$>$ Código de identificação do teste: código gerado automaticamente pelo AMC com 0 intuito de vincular o referido exemplar - na etapa 5 (a) de identificação do documento digitalizado como um exemplar gerado pelo próprio sistema - com as informações armazenadas em seu banco de dados. É importante esclarecer que os dados numéricos do enunciado (aqueles circulados) podem variar de uma versão do teste para outra; logo, o código de identificação do teste é importante para a etapa da correção automática da resposta numérica final, por exemplo;

$>$ Número na turma: é um número único de identificação individual que cada um dos estudantes recebe previamente (ID do aluno), e precisa preencher na folha de resposta de seu teste a fim de que o software AMC possa reconhecê-lo automaticamente, identificando e armazenando, em seu banco de dados, as informações pertinentes do referido estudante;

$>$ Nome: campo destinado à assinatura do estudante. Esse recurso ajuda o professor a identificar manualmente (por inspeção visual) a folha-resposta de um particular estudante, caso este estudante não tenha preenchido corretamente seu número na turma (ou tenha esquecido de fazê-lo), ou, ainda, caso ocorra alguma falha no sistema de identificação automática.

Na parte aberta de cada uma das questões dos testes, solicitávamos aos estudantes que descrevessem (justificassem) o processo de resolução pelo qual eles haviam obtido as respostas da parte numérica desses testes. Deste modo, esperávamos obter subsídios que nos permitissem analisar os raciocínios desenvolvidos pelos estudantes (ao menos os principais passos necessários para solucionar o problema proposto). E esperávamos, também, obter elementos que nos ajudassem verificar em que medida os estudantes estavam empregando adequadamente (ou não) os conceitos e procedimentos abordados nos referidos testes, no sentido de fornecer a eles um feedback mais rico do que simplesmente a correção da parte numérica dos referidos testes.

Entretanto, cabe destacar que a avaliação de questões abertas, como as mencionadas anteriormente, demanda um tempo considerável das tarefas do professor e não pode ser automatizada, sendo que a quantidade de tempo despendida nesse tipo de avaliação está diretamente relacionada com a complexidade da questão a ser analisada. No entanto, este processo pode tornar-se mais ágil caso o professor saiba antecipadamente se o aluno acertou a parte numérica. Por isso, neste estudo de caso, antes de corrigirmos as justificativas, os testes da turma passavam por um primeiro processamento no qual o sistema indicava o desempenho do aluno na parte numérica. Então, com base nessa pré-análise, executamos a avaliação da justificativa, isto é, da parte aberta da questão.

\subsection{Contexto de pesquisa}

O presente trabalho foi desenvolvido no contexto do ensino universitário na disciplina de Física Geral e Experimental I de cursos de Engenharia de uma universidade federal. Essa é uma das disciplinas em que esses estudantes têm os primeiros contatos com o ensino universitário (na maioria dos cursos envolvidos, é uma disciplina que se encontra na grade curricular do primeiro semestre, logo, na maioria dos casos, os alunos são praticamente oriundos do ensino médio). Nela, são abordados os conteúdos de Mecânica, tais como: cinemática, dinâmica, leis de conservação (energia, momento linear e angular) e rotações. A bibliografia básica da referi- 
da disciplina é um livro-texto de nível universitário, tal como: Halliday et al. (2008) ou Tipler e Mosca (2009). Ademais, destacamos que os problemas presentes nos finais dos capítulos do livro-texto adotado são utilizados como recurso didático - sendo empregados inclusive nas avaliações finais (cumulativas) - isto é, no contexto da referida disciplina, a resolução desse tipo de problema é uma prática didática comum tanto dos docentes quanto dos estudantes.

\subsection{Resultados obtidos com o uso do AMC}

Com a finalidade de analisarmos as potencialidades de uso do AMC, registrarmos o tempo utilizado nas etapas de correção e elaboração do feedback dos testes que implementamos, registrando, para isso, os tempos empregados para executar cada um dos passos diretamente relacionados a essas duas etapas. Os dados coletados com a avaliação de seis testes presenciais estão representados no Quadro 1.

Quadro 1 - Tempo usado para executar cada uma das etapas do processo de avaliação dos testes

\begin{tabular}{|c|c|c|c|c|c|c|c|}
\hline & $\mathrm{T} 1$ & $\mathrm{~T} 2$ & T3 & T4 & T5 & T6 & $\begin{array}{l}\text { Tempo médio por } \\
\text { teste }\end{array}$ \\
\hline $\begin{array}{l}\text { Número de testes (cada teste continha uma } \\
\text { questão) }\end{array}$ & 55 & 60 & 53 & 54 & 52 & 45 & - \\
\hline (4) tempo de digitalização (min) & 21,20 & 19,10 & 19,27 & 17,00 & 19,00 & 14,32 & 0,36 \\
\hline (5) tempo de processamento ( $\min$ ) & 6,18 & 9,30 & 6,38 & 6,40 & 7,00 & 6,13 & 0,13 \\
\hline $\begin{array}{l}\text { Tempo de avaliação não automática da } \\
\text { justificativa (min) }\end{array}$ & 75,00 & 57,30 & 126,00 & 39,00 & 38,00 & 30,00 & 1,13 \\
\hline $\begin{array}{l}\text { Tempo médio total de avaliação por teste } \\
(\mathrm{min})\end{array}$ & 1,86 & 1,43 & 2,86 & 1,15 & 1,23 & 1,21 & 1,62 \\
\hline
\end{tabular}

Fonte: elaborado pelos autores

Com base nos dados mostrados no Quadro 1 acima, apontamos as seguintes principais considerações:

$>$ Cada teste continha apenas uma questão (problema), mas o tempo de digitalização leva em conta, em geral, apenas o número de folhas-resposta. Caso uma folha-resposta tenha mais do que uma questão, o tempo empregado será o mesmo, o que pode ser considerado como uma vantagem de se utilizar o AMC;

$>$ O tempo médio de digitalização de cada folha-resposta foi cerca de 0,36 minutos $(21,6$ s). Cabe destacar que, embora a digitalização tenha sido realizada manualmente, esse tempo está relacionado com o desempenho do equipamento empregado na digitalização das folhas-resposta. Ou seja, se tivéssemos utilizado uma máquina que realizasse essa tarefa automaticamente, esse tempo teria sido reduzido consideravelmente (isso implica que o tempo de operação da máquina é praticamente desprezado, quando comparado ao do equipamento);

$>$ O tempo médio de processamento, por teste, foi de aproximadamente 0,13 minutos $(7,8 \mathrm{~s})$, o qual é praticamente irrelevante. Além disso, essa etapa traz as seguintes vantagens: (i) realizar uma pré-análise das resoluções dos alunos; e (ii) diminuir o risco de erro humano na etapa de correção (e atribuição de nota) da parte numérica da solução desses alunos;

$>$ O tempo médio de avaliação das justificativas, por teste, foi de 1,13 minutos. Embora essa etapa da avaliação tenha sido executada manualmente (isto é, pelo 
docente), ela foi bastante facilitada pela pré-análise executada na etapa de processamento, descrita no item anterior. Por outro lado, caso o professor opte por considerar apenas a parte numérica das questões, essa etapa não seria realizada. Consequentemente, o tempo de correção dos testes seria reduzido drasticamente.

$>$ Por fim, o AMC oferece a potencialidade de enviar automaticamente o feedback personalizado (folha-resposta avaliada, editada e comentada via editor de imagem) para o e-mail de cada um dos alunos. Ou seja, cada estudante recebe, em seu email pessoal, somente o seu teste corrigido (com a nota e comentários pertinentes).

\section{Considerações finais}

Neste trabalho, argumentamos que a realização de um processo avaliativo contínuo (ao longo do desenvolvimento de toda a disciplina) - que forneça feedback rápidos e frequentes para os estudantes - é mais produtivo do que somente a realização de avaliações pontuais e cumulativas, tanto para os professores quanto para os alunos. Porém, também reconhecemos que, no contexto atual - em que a quantidade de estudantes atendidos nas disciplinas universitárias de Física Básica vem aumentado consideravelmente (MELLO, 2015) - a realização de um processo avaliativo como esse torna-se pouco viável, uma vez que o processo de correção e feedback desse tipo de avaliação é bastante complexo e trabalhoso para o docente, sobrecarregando-o (OTSUKA e DA ROCHA, 2005).

Assim, argumentamos ainda que um passo importante no sentido de viabilizar esse tipo de avaliação é dinamizar essa etapa de correção e feedback. Uma das possíveis alternativas para essa dinamização é empregar instrumentos avaliativos compostos por problemas aliados a um software de correção automática, tal como o AMC. Por isso, neste trabalho, tínhamos por objetivo caracterizar as potencialidades desse software no sentido de dinamizar o processo de correção e feedback de provas e testes desse tipo.

Como principais potencialidades do software AMC, apontamos: (i) correção automática da resposta numérica final ou da alternativa correta; (ii) possibilidade variar os dados numéricos do enunciado de uma mesma questão (parametrizá-la), bem como as respostas numéricas finais, individualizando-as; (iii) possibilidade de criar um banco de questões que podem ser reutilizadas e/ou compartilhadas; (iv) possibilidade de variar a ordem das questões de uma prova ou teste, criando diversas versões deles; ( $v$ ) possibilidade de ajustar uma margem de erro para essa resposta numérica final; (vi) redução do risco de erro humano na correção numérica; e (vii) possibilidade de enviar o resultado da correção diretamente para o e-mail do aluno.

Cabe destacar, ainda, que os resultados encontrados nessa contribuição específica de aplicação do software AMC, em duas turmas, não tencionam ser generalizados para qualquer contexto de forma imediata. Em outras palavras, destacamos a necessidade de levar esse instrumento a outras realidades, como, por exemplo, em escolas da educação básica ou, mesmo, para outras disciplinas básicas de cursos universitárias; aumentando, dessa forma, as variáveis que possibilitem uma análise mais global da utilização dessa ferramenta em processos avaliativos formativos.

Por fim, sublinhamos que, em consequência das potencialidades listadas anteriormente, um dos principais impactos no ensino, viabilizado pelo emprego do software AMC é dinamizar a etapa de correção e feedback de testes e provas que utilizam questões baseadas em resolução de problemas para avaliar a aprendizagem dos estudantes. Ou seja, essa ferramenta é uma alternativa útil no sentido de viabilizar a realização de um processo avaliativo contínuo - que se dá ao longo do desenvolvimento de toda a disciplina -, permitindo fornecer feedback rápidos e frequentes para os estudantes, em particular no contexto de turmas compostas por um grande número de estudantes. 


\section{Referências}

ALVES, J.; SAUERWEIN, R. A.; PASTORIO, D. P. A resolução de problemas parametrizados: uma estratégia para estimular a aprendizagem colaborativa. Revista de Enseñanza de la Física, v. 29, n. extra, p. 113 $119,2017$.

CLEMENT, L.; TERRAZZAN, E. A. Resolução de problemas de lápis e papel numa abordagem investigativa. Experiências em Ensino de Ciências, v.7, n. 2, p. 98-116, 2012.

HALLIDAY, D., RESNICK, R. WALKER, J. Fundamentos de Física: Mecânica. Rio de Janeiro: Livros Técnicos e Científicos Editora S.A., 2008.

MAXIMO, L. F.; RAABE, A. L. A.; BARONE, D. A. C. Avaliação formativa assistida por computador no ensino a distância. RENOTE Revista Novas Tecnologias na Educação, v.5, n. 1, p. 1-10, 2007.

MELLO, B. A. Aumento na quantidade de alunos em disciplinas básicas: Como obter vantagens dessa realidade universitária. Revista Brasileira de Ensino de Física, v. 37, n. 3, p. 1-9, 2015.

OTSUKA, J. L.; FERREIRA, T. B.; LACHI, R. L., DA ROCHA, H. V. Um modelo de suporte à avaliação formativa no ambiente TelEduc. Revista Brasileira de Informática na Educação, v. 11, n. 2, p. 100-110, 2003.

OTSUKA, J. L.; DA ROCHA, H. V. Avaliação formativa em ambientes EaD: uma proposta de suporte tecnológico e conceitual. Revista Brasileira de Informática na Educação, v. 13, n. 2, p. 33-41, 2005.

PÉREZ-BENEDITO, J. L.; ARAGÓN, E. Q.; ALRIOLS, J. A.; MEDIC, L. Optical mark recognition in student continuous assessment. Revista Iberoamericana de Tecnologias del Aprendizaje, v. 9, n. 4, p. 133-138, 2014.

RAVITZ, J. CILT2000: using technology to support ongoing formative assessment in the classroom. Journal of Science Education and Technology, v. 11, n.3, p. 293-296, 2002.

TIPLER, P. A. MOSCA, G. Física para cientistas e engenheiros: Mecânica, Oscilações e Ondas, Termodinâmica. Rio de Janeiro: Livros Técnicos e Científicos Editora S.A., 2009.

VITTURINI, M.; BENEDETTI, L.; SEÑAS, P. Una nueva herramienta de evaluación basada en Filtros de Corrección Automática (FCA). Revista Iberoamericana de Tecnología en Educación y Educación en Tecnología.

n.6, p. 43-51, 2011.

Recebido em junho de 2018

Aprovado para publicação em dezembro de 2018

\section{Ricardo Andreas Sauerwein}

Professor adjunto do Departamento de Física - Universidade Federal de Santa Maria - UFSM, Brasil, rsauer.ufsm@gmail.com

\section{Josemar Alves}

Professor temporário do Departamento de Física - Universidade Federal de Santa Maria - UFSM, Brasil, josemarfis@gmail.com

\section{Dioni Paulo Pastorio}

Professor adjunto A - Universidade Federal do Rio Grande do Sul - UFRGS, Brasil, dionipaulopastorio@gmail.com 